\title{
Molecular Characterization of African Swine Fever Virus, China, 2018
}

\section{Shengqiang Ge, ${ }^{1}$ Jinming $\mathrm{Li},{ }^{1}$ Xiaoxu Fan, ${ }^{1}$ Fuxiao Liu, ${ }^{1}$ Lin Li, ${ }^{1}$ Qinghua Wang, Weijie Ren, Jingyue Bao, Chunju Liu, Hua Wang, Yutian Liu, Yongqiang Zhang, Tiangang $\mathrm{Xu}$, Xiaodong Wu, Zhiliang Wang}

Author affiliation: China Animal Health and Epidemiology Center, Qingdao, China

DOI: https://doi.org/10.3201/eid2411.181274

On August 3, 2018, an outbreak of African swine fever in pigs was reported in China. We subjected a virus from an African swine fever-positive pig sample to phylogenetic analysis. This analysis showed that the causative strain belonged to the p72 genotype II and CD2v serogroup 8 .

\begin{abstract}
A frican swine fever (ASF) is a disease that is reportable to the World Health Organisation for Animal Health. This disease causes high fever, hemorrhages, ataxia, and severe depression in domestic pigs and has mortality rates approaching $100 \%$. Its causative agent is African swine fever virus (ASFV; family Asfarviridae, genus Asfivirus), a large, enveloped, double-stranded DNA virus (1). ASF was first described in Kenya in 1921, and was introduced into the Republic of Georgia in 2007, after which it spread into other countries in eastern Europe, including Russia (2007), Ukraine (2012), Belarus (2013), Lithuania (2014), Estonia (2014), Poland (2014), Latvia (2014), Romania (2017), the Czech Republic (2017), and Hungary (2018).

During July 1-August 1, 2018, a total of 47 of 383 pigs died on a farm in the Shenbei District of Shenyang, Liaoning Province, China. Postmortem analysis performed by local veterinarians showed an ASF-typical lesion in pig spleens that were extremely swollen and severely necrotic. Other pathologic changes included hemorrhages in tonsils and lungs, marbled lesions in mandibular and mesenteric lymph nodes, and diffuse hemorrhages in a large part of gastric serosa.

We collected samples from 2 dead pigs and 6 live pigs on this farm and sent these samples to our Biosafety Level 3 laboratory for confirmation of ASFV infection. We performed a real-time PCR for ASFV as recommended by the World Health Organisation for Animal Health protocol. Results confirmed ASFV infections in China (2).

After confirmation of ASFV infection by our laboratory, we used nucleic acid extracts from an ASFV-infected
\end{abstract}

${ }^{1}$ These authors contributed equally to this article. sample for conventional PCR amplification with 3 pairs of primers. We amplified 3 gene fragments: a partial gene fragment of the $\mathrm{B} 646 \mathrm{~L}$ gene encoding the $\mathrm{p} 72$ capsid protein (3), a fragment of the EP402R gene encoding the CD2v protein (4), and a tandem repeat sequence (TRS) located between the I73R and I329L genes (5).

We subjected 3 amplified products to nucleotide sequencing and deposited the resulting sequences in GenBank (accession nos. MH722357, MH735142, and MH735144). We used the p72 fragment sequence for phylogenetic analysis of the genotype, and the $\mathrm{CD} 2 \mathrm{v}$ fragment sequence for phylogenetic analysis of the serogroup (6). We constructed 2 phylogenetic trees by using MEGA 5.0 software (https://www.megasoftware.net/). These trees showed that the causative strain (China 2018/1) in this study belonged to p72 genotype II (Figure, panel A) and to CD2v serogroup 8 (Figure, panel B).

Genotype identification of ASFV often depends on partial p72 gene characterization (3). During ASF outbreaks, this genotyping approach can be used to identify possible origins of viruses and differentiate them from closely related strains (7). In this study, we classified China 2018/1 as genotype II (Figure, panel A), the sequences we obtained had extremely high homology with those of other genotype II strains, therefore suggesting the origin of China 2018/1 from a homogenotypic strain.

In addition to conventional genetic typing, serologic typing is another method for classifying ASFVs on the basis of hemadsorption inhibition (HAI). Eight ASFV serogroups have been identified (6). Moreover, HAI typing places ASFV into discrete serogroups not necessarily resolved by the p72based genetic typing. For example, serogroup 1, 2, and 4 strains can be simultaneously classified as having the P72 genotype I (7). In this study, we found that China 2018/1 belonged to serogroup 8 as determined by phylogenetic analysis, suggesting the same HAI characteristics as those for other strains in the homoserogroup (Figure, panel B).

We compared Georgia 2007/1, which is representative of genotype II, with China 2018/1. China 2018/1 had a 10bp additional fragment (5'-GGAATATATA-3') that was inserted into the TRS between the I73R and I329L genes and was identical to those of the Bel13/Grodno, Ukr12/Zapo, Lt14/1490, Lt14/1482, Pol14/Sz, and Pol14/Krus strains (5).

ASF causes devastating socioeconomic consequences in the global pig industry, especially for countries with large-scale pig production and pork consumption. After the confirmation of ASF outbreak in China in August 2018, we characterized the causative strain, China 2018/1, by phylogenetic comparison with previous strains. We classified this new strain as having the p72 genotype II and $100 \%$ p 72 sequence identity with several strains from eastern Europe and Africa, such as Bel13/Grodno, Voronezh 2016, Mal 2011/01, and ZIM/2015/01. 


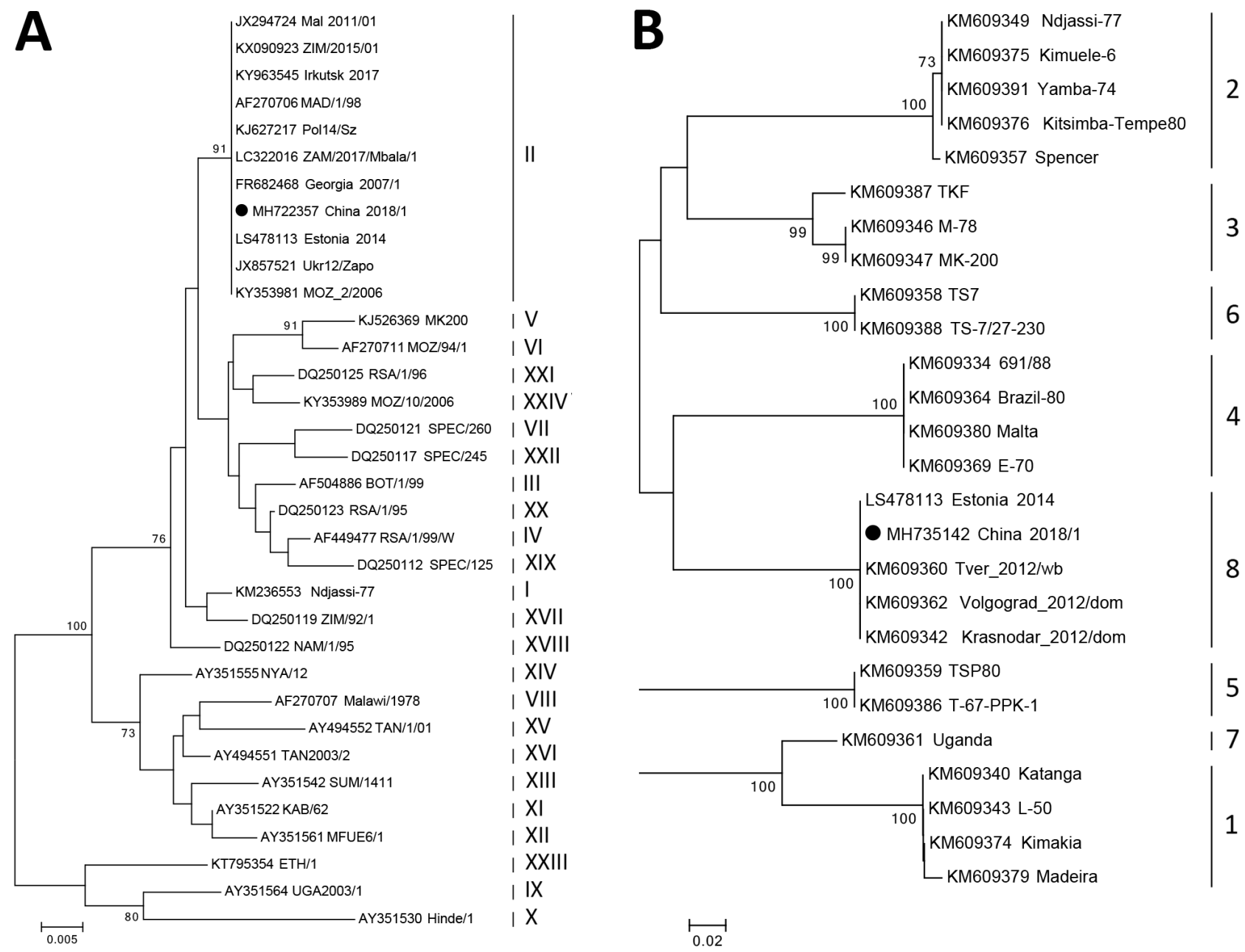

Figure. Phylogenetic analysis of a causative virus strain (China 2018/1) of an African swine fever outbreak, China, 2018 . A) p72 genotype; B) CD2v serogroup. The neighbor-joining method and Kimura 2-parameter model were used for construction of phylogenetic trees in MEGA 5.0 software (https://www.megasoftware.net/). Numbers along branches indicate bootstrap values $>70 \%$ (1,000 replicates). Black circles indicate causative virus from this study. Roman numerals to the right in panel A indicate p72 genotypes. Numbers to the right in panel B indicate CD2v serogroups. GenBank accession numbers are provided for all sequences. Scale bars indicate nucleotide substitutions per site.

On the basis of serologic typing, we found that China 2018/1 belonged to the CD2v serogroup 8. In addition, this strain had a 10-bp additional fragment (5'-GGAATATATA-3') in its TRS when compared with others in the homogenotype.

Because ASFV is a large DNA virus with a stable genome, it is hardly unexpected that the sequence of China 2018/1 had extremely high homology with sequences in genotype II over a wide geographic area. However, it remains to be elucidated from where China 2018/1 was introduced into China.

\section{Acknowledgments}

We thank our colleagues for assistance in collecting and transporting clinical samples.

This study was supported by the National Project for Prevention and Control of Transboundary Animal Diseases (grant no. 2017YFD0501800), the National Key Research and Development Program for the 13th Five-Year Plan, and the Ministry of Science and Technology, China. 


\section{About the Author}

Dr. Ge is a virologist at the National Research Center for Exotic Animal Diseases, Animal Health and Epidemiology Center, Qingdao, China. His primary research interest is prevention and control of exotic animal diseases.

\section{References}

1. Alonso C, Borca M, Dixon L, Revilla Y, Rodriguez F, Escribano JM; ICTV Report Consortium. ICTV virus taxonomy profile: Asfarviridae. J Gen Virol. 2018;99:613-4. http://dx.doi.org/ 10.1099/jgv.0.001049

2. Wang Q, Ren W, Bao J, Ge S, Li J, Li L, et al. The first outbreak of African swine fever was confirmed in China [in Chinese]. Journal of China Animal Health Inspection. 2018;35:1-5.

3. Bastos AD, Penrith ML, Crucière C, Edrich JL, Hutchings G, Roger F, et al. Genotyping field strains of African swine fever virus by partial p72 gene characterisation. Arch Virol. 2003; 148:693-706. http://dx.doi.org/10.1007/s00705-002-0946-8

4. Sanna G, Dei Giudici S, Bacciu D, Angioi PP, Giammarioli M, De Mia GM, et al. Improved strategy for molecular characterization of African swine fever viruses from Sardinia, based on analysis of p30, CD2V and I73R/I329L variable regions. Transbound Emerg Dis. 2017;64:1280-6.

http://dx.doi.org/10.1111/tbed.12504

5. Gallardo C, Fernández-Pinero J, Pelayo V, Gazaev I, Markowska-Daniel I, Pridotkas G, et al. Genetic variation among African swine fever genotype II viruses, eastern and central Europe. Emerg Infect Dis. 2014;20:1544-7. http://dx.doi.org/ 10.3201/eid2009.140554

6. Malogolovkin A, Burmakina G, Tulman ER, Delhon G, Diel DG, Salnikov N, et al. African swine fever virus CD2v and C-type lectin gene loci mediate serological specificity. J Gen Virol. 2015; 96:866-73. http://dx.doi.org/10.1099/jgv.0.000024

7. Malogolovkin A, Burmakina G, Titov I, Sereda A, Gogin A, Baryshnikova E, et al. Comparative analysis of African swine fever virus genotypes and serogroups. Emerg Infect Dis. 2015;21:312-5. http://dx.doi.org/10.3201/eid2102.140649

Address for correspondence: Xiaodong Wu or Zhiliang Wang, National Research Center for Exotic Animal Diseases, China Animal Health and Epidemiology Center, 369 Nanjing Rd, Qingdao, Shandong 266032, China; emails: wuxiaodong@cahec.cn or wangzhiliang@cahec.cn

\section{Familial Transmission of emm12 Group A Streptococcus}

\section{Rachel Mearkle, Sooria Balasegaram, Shiranee Sriskandan, Vicki Chalker, Theresa Lamagni}

Author affilliations: Public Health England, Chilton, UK (R. Mearkle); Public Health England, London, UK (S. Balasegaram, V. Chalker, T. Lamagni); Imperial College, London, UK (S. Sriskandan)

DOI: https://doi.org/10.3201/eid2411.171743

To the Editor: We read with interest the recent research letter by Duployez et al. describing a cluster of invasive group A Streptococcus (iGAS) infections in a cohabiting couple in their $60 \mathrm{~s}$ (1). The report illustrates the increased risk of infection for persons living in a household with someone with iGAS infection. We write to draw readers' attention to our recent study, which adds to the body of evidence on the risk of household transmission of iGAS (2).

Population-based studies from Australia, Canada, the United Kingdom, and the United States, based on 13 household clusters, assessed the risk of transmitting iGAS infection through household contact (3). We identified an additional 24 household clusters in England using addresses captured through national surveillance in 2009 and 2011-2013. For all 12 clusters in which emm typing was performed on both patients, results were the same for both. All secondary cases occurred within 1 month of the index case (median 2 days). Among contacts, the 30day incidence rate was 4,520/100,000 person-years, 1,940 times higher than the background incidence $(2.34 / 100,000$ person-years). Spouses and partners $\geq 75$ years of age (6 pairs) were at particularly high risk for developing infection; incidence was estimated at $15,000(95 \%$ CI 5,510 $32,650) / 100,000$ person-years, 1,650 times higher than the background risk in this age group $(9.09 / 100,000,95 \%$ CI $5,510-32,650)$. These data resulted in an estimated number needed to treat of $82(46-417)$.

Duployez's article also highlights differences between countries in policies for antimicrobial chemoprophylaxis. National guidance for public health management of community iGAS infection is being revised in the United Kingdom; oral penicillin $\mathrm{V}$ is currently recommended as the first choice for chemoprophylaxis (4). However, questions remain about the efficacy of chemoprophylaxis and the practicalities of timely administration to benefit others in a household, given that $38 \%$ of pairs were co-primary cases or had only 1 day between initial and subsequent infections.

\section{References}

1. Duployez C, Vachée A, Robineau O, Giraud F, Deny A, Senneville E, et al. Familial transmission of emm 12 group A Streptococcus. Emerg Infect Dis. 2017;23:1745-6. http://dx.doi.org/ 10.3201/eid2310.170343 